\title{
MicroRNA-378 promotes myogenic differentiation by targeting BMP4
}

\author{
HUIMING JU ${ }^{1,2^{*}}$, YUEFEI YANG ${ }^{1,2^{*}}$, ANZHI SHENG ${ }^{1,2}$ and YUYU QI ${ }^{1,2}$ \\ ${ }^{1}$ College of Veterinary Medicine, Yangzhou University; \\ ${ }^{2}$ Jiangsu Co-innovation Center for Prevention and Control of Important Animal Infectious Diseases and Zoonoses, \\ Yangzhou, Jiangsu 225009, P.R. China
}

Received February 12, 2015; Accepted December 1, 2015

DOI: $10.3892 / \mathrm{mmr} .2016 .4764$

\begin{abstract}
MicroRNA-378 (miRNA-378) has been reported to have a crucial role in skeletal muscle differentiation; however, the underlying mechanisms have largely remained to be elucidated. The present study employed high-throughput RNA sequencing to investigate the transcriptome following transfection of miRNA-378 mimics or control RNAs into $\mathrm{C} 2 \mathrm{C} 12$ myoblast cells. By sequencing and annotation, 2,802 transcripts that were changed by $>1.5$ fold were obtained and then subjected to signaling pathway enrichment and gene ontology analysis. Eight genes associated with development were subsequently selected for validation by quantitative qPCR, the results of which were highly consistent with those of the high-throughput RNA sequencing. The protein levels of bone morphogenetic protein 4 (BMP4), which was among the differentially expressed genes, were decreased following ectopic expression of miRNA-378. BMP4 was further confirmed to be a direct target of miRNA-378 by using a dual luciferase assay. Finally, treatment with miRNA-378 or small interfering RNA against BMP4 induced myogenic differentiation in $\mathrm{C} 2 \mathrm{C} 12$ cells. In conclusion, the present study suggested that miRNA-378 is critical for the promotion of myoblast differentiation by targeting BMP4.
\end{abstract}

\section{Introduction}

The development of skeletal muscle is controlled by an evolutionarily conserved transcription factor network involving in the regulation of genes associated with muscle differentiation, contractility and growth $(1,2)$. Myocyte enhancer factor-2

Correspondence to: Dr Huiming Ju, College of Veterinary Medicine, Yangzhou University, 12 Wenhui East Road, Yangzhou, Jiangsu 225009, P.R. China

E-mail: huimingju1@126.com

${ }^{*}$ Contributed equally

Key words: microRNA-378, differentiation, bone morphogenetic protein 4
(MEF2) is the longest known myogenic transcription factor, and most muscle-associated genes are directly activated by MEF2 through combined interaction with other transcription factors (3). Studies have demonstrated that, in addition to activating genes involved in muscle contraction and differentiation, myogenic transcription factors regulate the expression of a number of conserved microRNAs (miRNAs) that function to 'fine-tune' the output of transcriptional networks, leading to precise cellular responses to pathological, physiological and developmental signals (4). As individual miRNAs can regulate hundreds of mRNAs and individual mRNAs are in turn targeted by numerous miRNAs, miRNAs add complexity and accuracy to the regulation of the core muscle transcriptional network (5).

miRNAs are endogenous, small, non-coding RNAs of $\sim 22 \mathrm{nt}$ in length that have emerged as powerful negative regulators of gene expression (6). By selectively repressing the activity of the 3'-untranslated region (3'-UTR) of target mRNAs, miRNAs confer appropriate timing and robustness in differentiation programmes. A set of miRNAs, including miRNA-1, miRNA-133, miRNA-206, miRNA-208, miRNA-486 and miRNA-499, have been identified to be highly enriched in skeletal and/or cardiac muscle (7). Certain key myogenic regulatory factors (MRFs), including myogenin and myogenic differentiation 1 (MyoD1), are known to regulate these miRNAs $(8,9)$. Several miRNAs that are normally induced during myogenic differentiation can initiate the myogenic program in $\mathrm{C} 2 \mathrm{C} 12$ myoblasts, even in the presence of high serum $(10,11)$. miRNA-378, a cardiac-enriched miRNA, has been shown to directly target insulin-like growth factor 1 receptor and regulate post-natal cardiac re-modeling (12). However, the functions of miRNA-378 in the differentiation of skeletal muscle have largely remained elusive.

To reveal the underlying mechanisms of the roles of miRNA-378 in myoblast differentiation, the present study employed a gain-of-function approach by transfecting miRNA-378 mimics into C2C12 myoblast cells and analyzing the resulting gene expression profiles by means of genome-wide mRNA deep sequencing. The present study demonstrated that miRNA-378 promoted myoblast differentiation by targeting the bone morphogenetic protein 4 (BMP4) gene. 


\section{Materials and methods}

Cell culture. C2C12 myoblasts (Shanghai Cell Bank of the Chinese Academy of Sciences, Shanghai, China) were cultured in Dulbecco's modified Eagle's medium (DMEM; Gibco; Thermo Fisher Scientific, Inc., Waltham, MA, USA) containing 20\% fetal bovine serum (Gibco; Thermo Fisher Scientific,Inc.) and $1 \%$ penicillin/streptomycin (Gibco; Thermo Fisher Scientific, Inc.) at $37^{\circ} \mathrm{C}$ in a humidified atmosphere with $5 \% \mathrm{CO}_{2}$. Transfection with miRNA mimics or plasmids was performed using Lipofectamine 2000 (Invitrogen; Thermo Fisher Scientific, Inc.) according to the manufacturer's protocols.

cDNA library construction. $\mathrm{C} 2 \mathrm{C} 12$ cells transfected with miRNA-378 mimics or control RNA (synthesized by Suzhou GenePharma Co., Ltd., Suzhou, China) were collected, and total RNA was then extracted using TRIzol reagent (Invitrogen). The sequences of the miRNA-378 mimics are as follows: Sense, 5'-CUCCUGACUCCAGGUCCUGUGU-3' and antisense, 5'-ACACAGGACCUGGAGUCAGGAG-3'. The quality of the RNA was determined with a NanoDrop ND-1000 (Nanodrop Technologies, Wilmington, DE, USA), and detected with $1 \%$ agarose gels (Beyotime Institute of Biotechnology, Haimen, China). Total RNA (10 $\mu \mathrm{g})$ was used for sequencing with an Illumina Hiseq2000 (Illumina, San Diego, CA, USA). To construct a cDNA library, oligo (dT) beads (Qiagen $\mathrm{GmbH}$, Hilden, Germany) were used to isolate mRNA. The mRNA was digested by $10 \mathrm{U} / \mu \mathrm{l}$ EcoRI (Takara Biotechnology Co., Ltd., Dalian, China) into short fragments, and first-strand cDNA was synthesized using random hexamer primers (Takara Biotechnology Co., Ltd.). Following synthesis of the second-strand cDNA, the double-stranded cDNA was purified using a QIAquick PCR Extraction kit (Qiagen GmbH) following the manufacturer's instructions. The purified fragments were enriched by polymerase chain reaction (PCR) to generate a cDNA library (13) and were then sequenced with the IlluminaHiseq2000.

Data assembly and annotation. Raw data were generated by sequencing using the Illumina Hiseq2000. The data were assembled into transcripts, unigenes and contigs with Trinity de novo software (version 3.1; http://www.blast2go.com), excluding the low-quality sequences, adaptors, sequences with uncertain bases and sequences of $>50 \mathrm{bp}$. To identify differentially expressed genes between miRNA-378-transfected samples and control RNA-transfected samples, expression ratios were calculated using the Limma algorithm in $\mathrm{R}$, applying moderated t-tests. Benjamini-Hochberg correction was then used for correcting multiple hypothesis testing, in which the q-value was calculated for each P-value. Genes with an absolute $\log 2$ expression ratio of $>0.6$ between miRNA-378-transfected and control RNA-transfected group and a q-value of $<0.005$ were considered to be significant under the corresponding treatment conditions. In addition, to identify enriched Gene Ontology (GO) terms (http://www. geneontology.org) in the sets of differentially expressed genes, the Database for Annotation, Visualization and Integrated Discovery Bioinformatics Resource (14) was used, where $\mathrm{P}<0.01$ was considered to indicate a statistically significant difference. The Kyoto Encyclopedia of Genes and Genomes (KEGG) database (http://www.genome.jp/kegg/) was used to analyze the metabolic pathways of the genes.

Target gene prediction. Miranda databases (www.microrna. org/) and TargetScan version 4.0 (www.targetscan.org/) were employed to identify potential target genes of miRNA-378 that were downregulated in miRNA-378-transfected C2C12 cells compared with control RNA-transfected cells. Following identification of potential target genes, SigTerm software (version 7.0) was used to confirm the prediction results.

Plasmid construction. The BMP4 or transforming growth factor beta 2 (TGFB2) 3'-UTR sequences were PCR-amplified from $\mathrm{C} 2 \mathrm{C} 12$ genomic DNA and cloned into the pGL3-control vector (Promega Corporation, Madison, WI, USA). Amplification was conducted using primers from Sangon Biotech Co., Ltd., Shanghai, China) in a PTC240 PCR system (Bio-Rad Laboratories, Inc., Hercules, CA, USA). The reaction mixture was incubated at $95^{\circ} \mathrm{C}$ for $30 \mathrm{sec}$, followed by 30 cycles of $95^{\circ} \mathrm{C}$ for $30 \mathrm{sec}, 56^{\circ} \mathrm{C}$ for $30 \mathrm{sec}$ and $72^{\circ} \mathrm{C}$ for $60 \mathrm{sec}$. The products were sequenced to verify the results. Mutagenesis was performed by PCR (15), followed by DpnI (10 U/ $\mu 1$; Takara Biotechnology Co., Ltd.) digestion to remove parental template DNA and obtain the mutated sequences BMP4-mut or TGFB2-mut.

Reverse-transcription quantitative $(R T-q) P C R$. Cells were lysed and total RNA was extracted using TRIzol reagent (Invitrogen) following the manufacturer's instructions. cDNA synthesis was performed using the Superscript III first-strand synthesis system (Invitrogen). qPCR was then performed using SYBR Green PCR master mix in an ABI cycler (Thermo Fisher Scientific, Inc.). ABI 7300 software (Thermo Fisher Scientific, Inc.) was used for quantitative analysis.

Dual luciferase assay. To assess whether BMP4 or TGFB2 are direct targets of miRNA-378, firefly luciferase reporter vectors ( $2 \mathrm{ng}$; Promega Corporation) driven by fragments from the respective gene's 3'-UTR or their mutants together with miRNA-378 mimics and a Renilla luciferase control vector ( $1 \mathrm{ng}$ ) were co-transfected into $\mathrm{C} 2 \mathrm{C} 12$ cells according to the manufacturer's protocols. Experiments were analyzed using a dual-luciferase reporter assay system (Promega Corporation) following the manufacturer's protocols. The luciferase signal was quantified using a luminometer (Monolight 3020; BD Biosciences, Franklin Lakes, NJ, USA).

Western blot analysis. At two days following transfection with miRNA-378 mimics or control RNA (100 nM), C2C12 cells were lysed in radioimmunoprecipitation buffer [Beyotime Institute of Biotechnology; $150 \mathrm{mM}$ sodium chloride, $1 \%$ Nonidet P-40, $0.1 \%$ sodium dodecyl sulfate, $50 \mathrm{mM}$ Tris- $\mathrm{HCl}$ (pH 8.0) and $0.5 \%$ sodium deoxycholate] supplemented with $1 \%$ protease inhibitor cocktail stock solution (Roche Diagnostics $\mathrm{GmbH}$, Mannheim, Germany). Protein concentration was determined by BCA Protein assay kit (Beyotime Institute of Biotechnology). Proteins $(20 \mu \mathrm{g})$ were subjected to sodium dodecyl sulfate polyacrylamide gel electrophoresis (Beyotime Institute of Biotechnology). After electroblotting onto a 
A

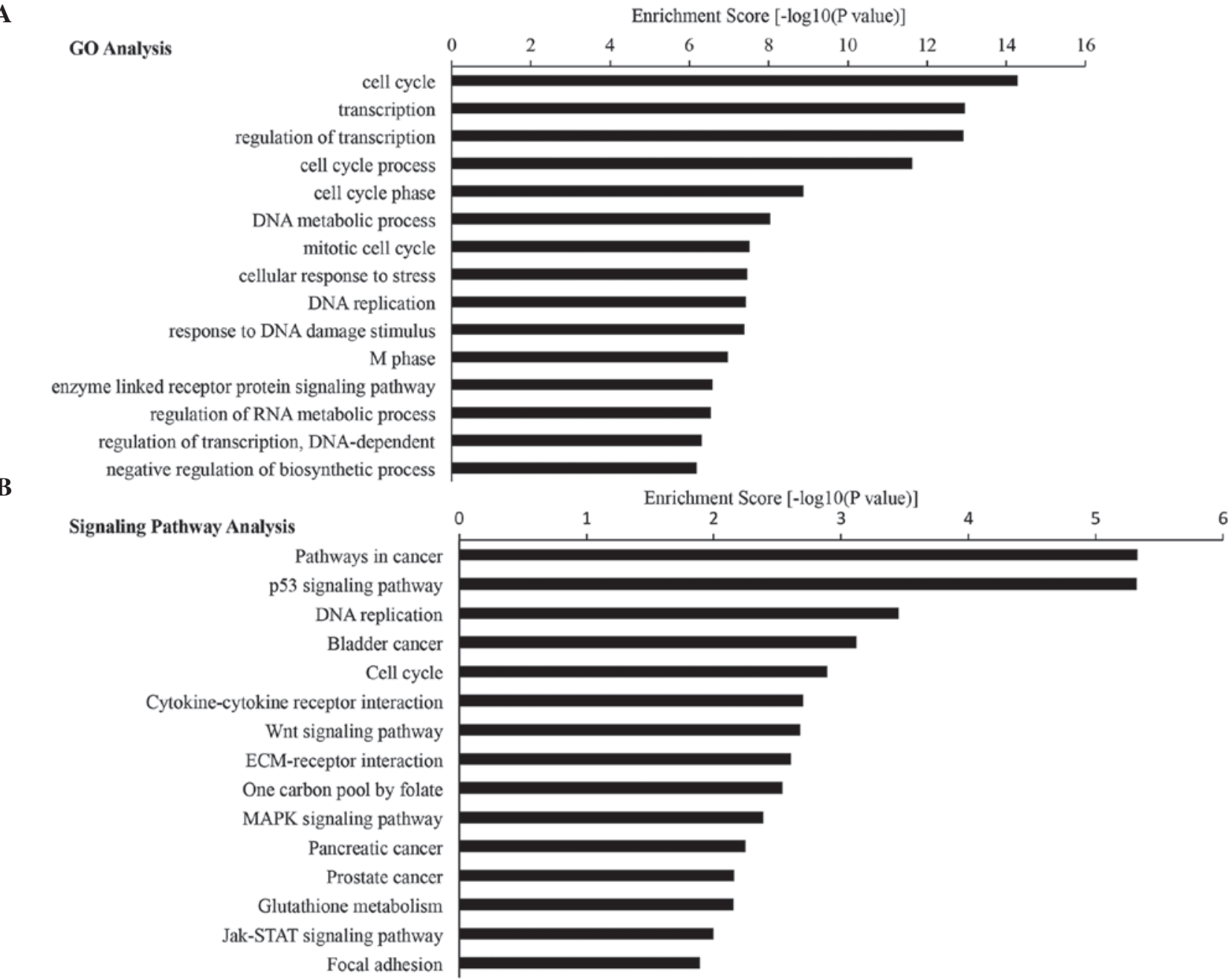

Figure 1. GO and signaling pathways of the downregulated genes. (A) Functional gene annotation of the differentially expressed genes according to the GO tool (http://www.geneontology.org). (B) Kyoto Encyclopedia of Genes and Genomes signaling pathway analysis for the differentially expressed genes (http://www.genome.jp/kegg/). GO, gene ontology. ECM, extracellular matrix; MAPK, mitogen-activated protein kinase; Jak, Janus kinase; STAT, signal transducer and enhancer of transcription.

polyvinylidene fluoride (PVDF) membrane (EMD Millipore, Billerica, MA, USA), Tris-buffered saline $/ 0.05 \%$ Tween-20 (TBST; Beyotime Institute of Biotechnology) containing 1\% skimmed milk was used to block non-specific binding sites. The PVDF membrane was then incubated with rabbit polyclonal anti-BMP4 (1:500 dilution; Abcam, Cambridge, MA, USA; cat. no. ab39973), rabbit polyclonal anti-TGFB2 (1:200 dilution; Abcam; cat. no. ab66043), rabbit polyclonal anti-MyoR (1:200 dilution; Santa Cruz Biotechnology, Inc., Dallas, TX, USA; cat. no. sc-366698), rabbit polyclonal anti-MyoD1 (1:200 dilution; Abcam; cat. no. ab64159), anti-MyHC (1:200 dilution; Abcam; cat. no. ab185967) or with rabbit monoclonal anti-glyceraldehyde 3-phosphate dehydrogenase (1:2,000 dilution; Sigma-Aldrich, St. Louis, MO, USA; cat. no. G9545) for $1 \mathrm{~h}$ at room temperature prior to washing three times for 10 min with TBST. The membrane was then incubated with horseradish peroxidase-conjugated goat anti-rabbit immunoglobulin $\mathrm{G}$ secondary antibodies (1:2,000 dilution; Abcam, cat. no. ab6721) for $1 \mathrm{~h}$ at room temperature. Signals were analyzed using enhanced chemiluminescence reagent, ECL Start (GE Healthcare, Little Chalfont, UK) and visualized with a Bio-Rad ChemiDoc MP system (Bio-Rad Laboratories, Inc.).
Small interfering RNA (siRNA) transfection. An siRNA against BMP4, termed si-BMP4 (Suzhou GenePharma Co., Ltd.) was used to endogenously inhibit BMP4. The sequences were as follows: Sense, 5'-AGGGCCAGGAAGAAGAAU AAUUUU-3' and antisense, 5'-AUUAUUCUUCUUCCU GGCCCUUUU-3'. Cells were transfected with $100 \mathrm{nM}$ of si-BMP4, miRNA-378 or negative control (NC) RNAs using Lipofectamine 2000 according to the manufacture's protocols. After $24 \mathrm{~h}$ transfection, the cells were harvested and subjected to the creatine kinase assay.

Creatine kinase (Ck) assay. Ck enzymatic activity was measured in cell lysates using the EnzyChrom Creatine Kinase assay kit (ECPK-100; BioAssay Systems LLC, Hayward, CA, USA) according to the manufacturer's protocol and as described previously (16). In brief, cells were washed twice with phosphate-buffered saline, lysed on ice for $10 \mathrm{~min}$ and scraped to remove cellular debris. The supernatant was then enriched by centrifuging at $8,000 \mathrm{x}$ g for $10 \mathrm{~min}$, and a 2.5 -fold volume of $\mathrm{H}_{2} \mathrm{O}$ was added, of which $10 \mu \mathrm{l}$ was used for $\mathrm{Ck}$ analysis. Evaluation was performed using the Bio-Rad BCA Protein assay kit (Bio-Rad Laboratories, Inc.). The absorbance 
A

B

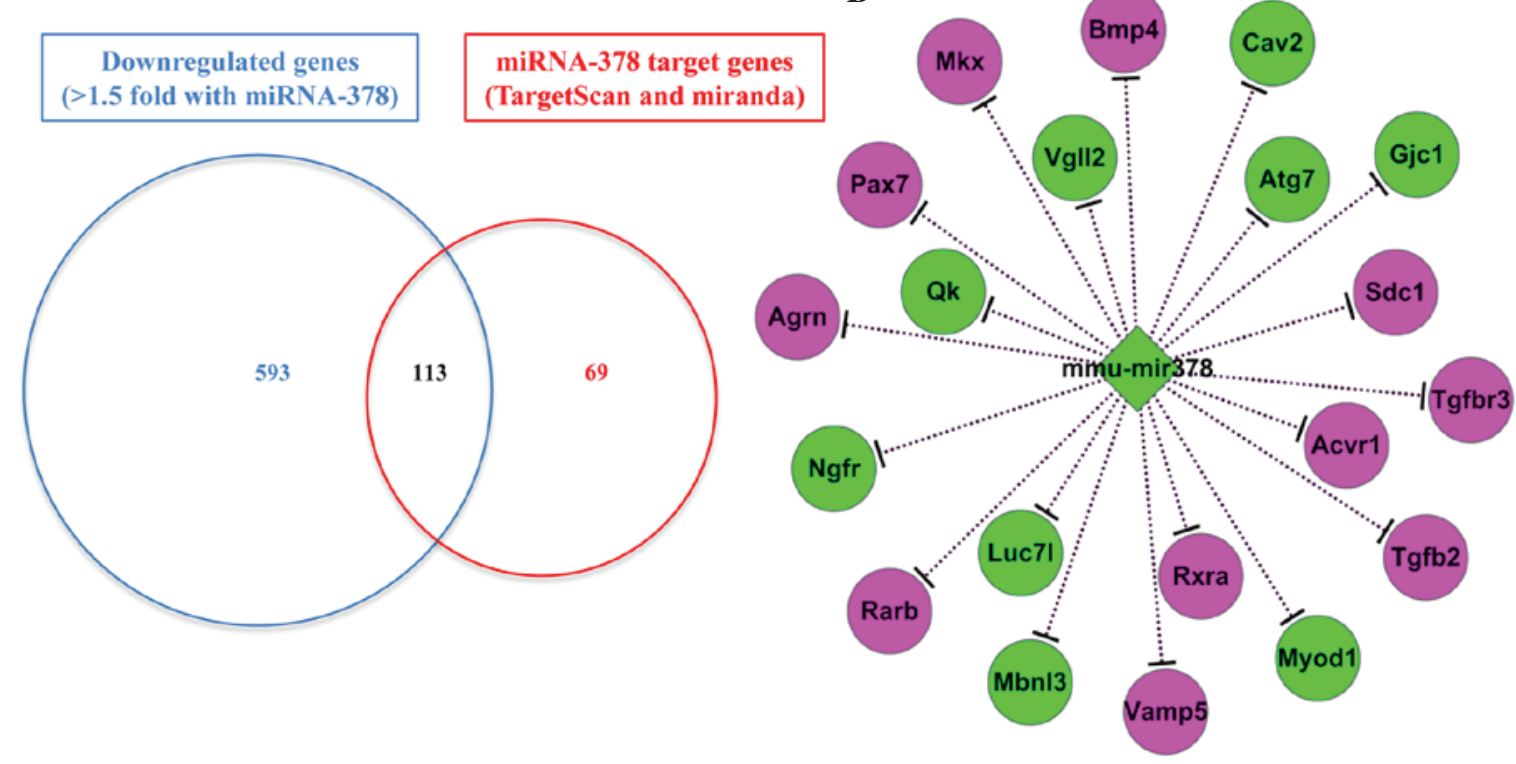

Figure 2. Transcriptome analysis the miRNA-378-regulated genes. (A) Venn diagram illustrating the association between the downregulated genes and predicted target genes by Targetscan and Miranda. (B) Network map illustrating the regulatory association between miRNA-378 and genes involved in development or differentiation. Downregulated genes are shown in magenta and upregulated genes in green. miRNA, microRNA.

was measured at $595 \mathrm{~nm}$ using a UV-1780 UV spectrophotometer (Shimadzu Corporation, Kyoto, Japan) and the results were expressed in arbitrary units with normalization to total protein content.

Statistical analysis. Values are expressed as the mean \pm standard deviation of at least three independent experiments. The two-tailed Student's $t$-test was used for performing statistical analysis in Excel software (version 2007). P<0.05 was considered to indicate a statistically significant difference.

\section{Results}

Transcriptome analysis for the assessment of aberrant genome-wide mRNA expression following miRNA-378 overexpression. To enhance the current understanding of the role of miRNA-378 in C2C12-cell differentiation, the present study performed a genome-wide gene expression analysis of miRNA-378-transfected cells in comparison with control RNA-transfected cells. A total of 2,802 genes that were differentially expressed by $>1.5$-fold following miRNA-378 treatment for $24 \mathrm{~h}$ were identified. To evaluate the potential functional significance of the differentially expressed genes, this set of genes was subjected to gene ontology (GO) pathway enrichment analysis. The results revealed a significant enrichment of a number of GO terms associated with the cell cycle (Fig. 1A). To further explore the canonical pathways involved, the genes were then subjected to signaling pathway enrichment analysis with the KEGG. As shown in Fig. 1B, the 15 most significantly upregulated pathways included the p53 signaling pathway and Wnt signaling pathway.

The 706 downregulated genes were then compared with the 182 predicted target genes of miRNA-378 obtained using the TargetScan and Miranda databases (Fig. 2A). A total of 113 candidate target genes of miRNA-378 were thereby identified, which were also significantly overrepresented in the downregulated gene sets obtained by SigTerm software (http://sigterms.sourceforge.net) analysis (17). The candidate genes included TGFB2, MKX, PAX7, RXRA and BMP4, which function in muscle development and muscle cell differentiation (10). Furthermore, of these candidate genes, the genes associated with development or differentiation were clustered as a regulatory network with miRNA-378, as illustrated in Fig. 2B generated by String software (version 9.0) (18). The interaction network showed that certain genes, including BMP4, RXRA, ACVR1 and SDC1, were potentially downregulated by miRNA-378, whereas other genes, including QK, ATG7, CAV2 and GJC1, were upregulated by miRNA-378.

BMP4 is a direct target of miRNA-378. To validate the results of the transcriptome sequencing, eight genes, GJC1, ATG7, QK, MYOD1, TGFB2, RARB, TGFBR3 and BMP4, were selected for further qPCR analysis, since the functions of these genes are involved in muscle differentiation or development. The PCR results demonstrated that the mRNA levels of four genes, GJC1, ATG7, QK and MYOD1, were significantly upregulated, while TGFB2 and BMP4 were markedly downregulated in miRNA-378-transfected cells compared with the negative control group. The mRNA expression of RARB and TGFBR3 was not markedly affected by miRNA-378 (Fig. 3A).

To further examine the potential targets of miRNA-378, western blot analysis of BMP4 and TGFB2 protein expression was performed in miRNA-378-transfected C2C12 cells. Transfection of miRNA-378 resulted in an obvious decrease in endogenous BMP4 protein, but had no effect on the protein levels of TGFB2 (Fig. 3B). These results indicated that miRNA-378 affected BMP4 expression at the mRNA as well as the protein level. To further validate whether miRNA-378 is a direct regulator of BMP4 or TGFB2, luciferase plasmids containing the BMP4 or TGFB2 3'-UTR downstream of the 
A
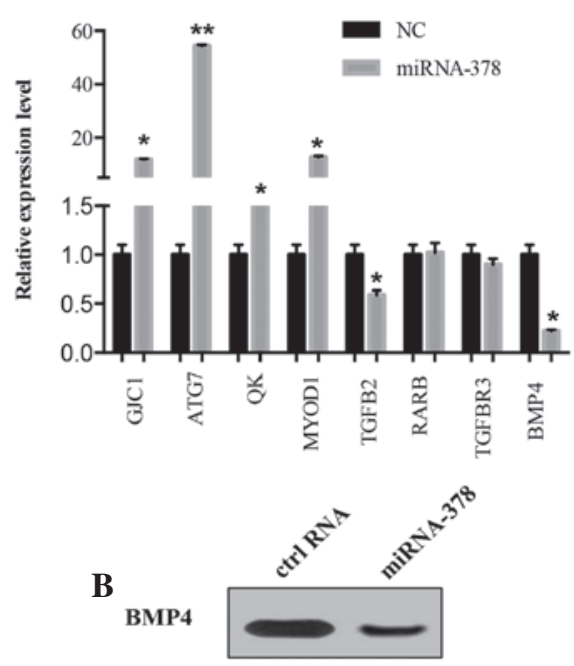

TGFB2

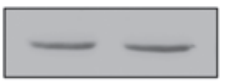

GAPDH

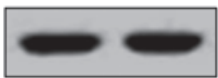

C

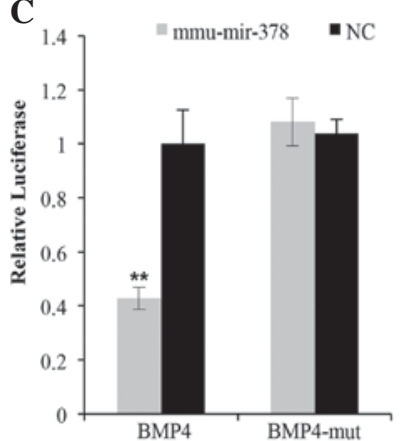

A
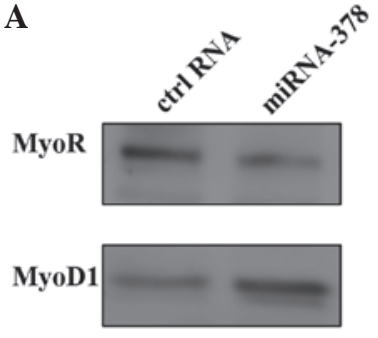

MyHC

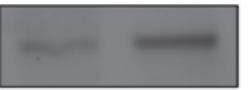

GAPDH

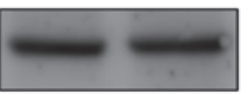

B

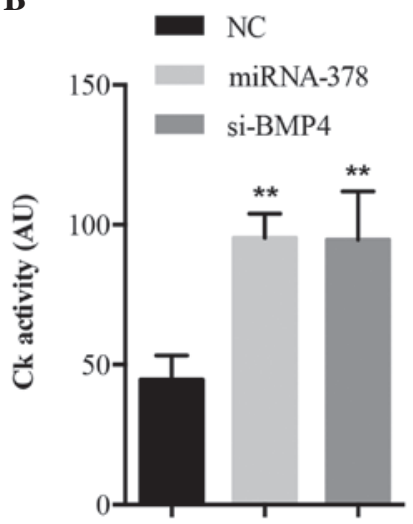

Figure 4. miRNA-378 promotes myogenic differentiation. (A) Western blot analysis of MyoR, MyoD1 and MyHC protein in the miRNA-378 mimics or control RNA-transfected $\mathrm{C} 2 \mathrm{C} 12$ cells. GAPDH served as protein loading control. Representative blots of three experiments are shown. (B) $\mathrm{C} 2 \mathrm{C} 12$ cells transfected with miRNA-378 mimics, si-BMP4 or control RNAs were analyzed for $\mathrm{Ck}$ activity. Values are expressed as the mean \pm standard deviation of three independent experiments. ${ }^{* *} \mathrm{P}<0.001$ vs. the NC group. miRNA, microRNA; GAPDH, glyceraldehyde 3-phosphate dehydrogenase; NC, negative control; siRNA, small interfering RNA; BMP4, bone morphogenetic protein 4; Ck, creatine kinase; MyoR, myogenic repressor; MyoD1, myogenic differentiation 1; MyHC, myosin heavy chain.

Effect of miRNA-378 on myogenic differentiation. Finally, the present study investigated the overall effect of miRNA-378 overexpression on the myogenic differentiation of $\mathrm{C} 2 \mathrm{C} 12$ by assessing the expression of a panel of differentiation biomarkers. As shown in Fig. 4A, C2C12 cells transfected with miRNA-378 mimics showed a significantly increased expression of myosin heavy chain (MyHC) and MyoD1 proteins, which are commonly detected in post-mitotic muscle cells and in committed proliferating myoblast cells, respectively, along with a concomitant decrease in the expression of myogenic repressor (MyoR), an inhibitor of myogenesis (10). Furthermore, the effect on myogenic differentiation was evaluated by comparing Ck activity in miRNA-378-transfected cells and control RNA-transfected cells. Ck activity in miRNA-378-transfected cells was significantly higher than that in control RNA-treated cells (Fig. 4B). The present study further examined whether knockdown of endogenous BMP4 was able to promote myogenic differentiation in $\mathrm{C} 2 \mathrm{C} 12$ cells. As shown in Fig. 4B, transfection of BMP4 siRNA and miRNA-378 resulted in an increase of $\mathrm{Ck}$ activity compared with the control group. This indicates that the downregulation of BMP4 mimics the function of miRNA-378 on myogenic differentiation of $\mathrm{C} 2 \mathrm{C} 12$ cells, further indicating that miRNA-378 regulates myogenic differentiation by downregulating BMP4.

luciferase gene were constructed and used in a dual luciferase assay with miRNA-378. The results showed that in miRNA-378-transfected cells, the luciferase activity of the BMP4 3'-UTR-driven vector was reduced by $\sim 60 \%$ compared with that in the negative control-transfected group; however, luciferase activity of the mutant luciferase reporter vector for BMP4 was not affected by miRNA-378 (Fig. 3C). This result confirmed that BMP4 is a direct target of miRNA-378. However, miRNA-378 did not affect the luciferase activity of the TGFB2 or TGFB2-mut reporter vectors, suggesting that miRNA-378 did not directly target the TGFB2 gene (Fig. 3D).

\section{Discussion}

The present study performed a genome-wide mRNA deep sequencing analysis to investigate the changes in mRNA expression in miRNA-378-transfected C2C12 cells and demonstrated that miRNA-378 promoted myogenic differentiation by directly targeting the BMP4 gene.

The process of muscle differentiation occurring in embryonic as well as adult muscle precursors is accompanied by the 
induction of a specific class of miRNAs, whose expression is driven by MRFs and other myogenic-dependent transcription factors $(8,19)$. Functionally, two different groups of miRNAs are found in muscle tissue: i) Non-muscle-specific miRNAs which are present in muscles, but are also expressed in other tissues (20) and ii) muscle-specific miRNAs, which are present in cardiac and skeletal muscle tissues in greater amounts compared with other tissues (20,21). Muscle-specific miRNAs were indeed shown to act on various levels in the regulation of muscle homoeostasis and differentiation, and their expression was found to be aberrant in certain muscular disorders, including Duchenne muscular dystrophy, myocardial infarction and other types of myopathy $(22,23)$. Among muscle-specific miRNAs, the miR-1/206 and miR-133 families, which originate from three different chromosomes (10), have been most extensively studied and have been shown to take multiple roles in the modulation of muscle differentiation (24). In addition, several non-muscle-specific miRNAs, including miRNA-24, miRNA-214 and miRNA-26a have been shown to participate in the differentiation process into muscle cells (25-27).

Studies have indicated that miRNA-378 is involved in skeletal muscle development $(28,29)$. In the $\mathrm{C} 2 \mathrm{C} 12$ myoblast cell line, upregulation of miRNA-378 has been shown to promote efficient myotube formation by repressing antagonists of differentiation, such as MyoR (29). Likewise, miRNA-378 expression in porcine longissimus muscles was shown to be implicated in the modulation of myogenesis, mainly with regard to fibre formation (28). Recently, miRNA-378 has been shown to induce skeletal muscle differentiation in the RH30 human alveolar rhabdomyosarcoma cell line (13). In accordance with these findings, the present study demonstrated that miRNA-378 promoted myogenic differentiation of $\mathrm{C} 2 \mathrm{C} 12$ cells via the BMP4 gene. Aberrant expression of specific myogenic biomarkers indicated the induction of myogenic differentiation. Transfection of miRNA-378 resulted in a significant increase of MyoD1 and MyHC protein and a decrease of MyoR protein. Furthermore, transfection of $\mathrm{C} 2 \mathrm{C} 12$ cells with siRNA against BMP4 mimicked the effect generated by miRNA-378, suggesting that miRNA-378 activated myogenic differentiation by targeting BMP4.

In conclusion, the present study demonstrated that miRNA-378 promoted myogenic differentiation by downregulating BMP4. These findings enhanced the current understanding of the biological mechanisms underlying muscle differentiation.

\section{Acknowledgements}

The present study was supported by the Priority Academic Program Development of Jiangsu Higher Education Institutions, Jiangsu Co-innovation Center for Prevention and Control of Important Animal Infectious Diseases and Zoonoses, and the National Natural Science Foundation of China (grant nos. 31101683 and 31272405 ).

\section{References}

1. Buckingham M: Skeletal muscle formation in vertebrates. Curr Opin Genet Dev 11: 440-448, 2001.

2. Buckingham M: Myogenic progenitor cells and skeletal myogenesis in vertebrates. Curr Opin Genet Dev 16: 525-532, 2006.
3. Satou Y and Satoh N: Gene regulatory networks for the development and evolution of the chordate heart. Genes Dev 20: 2634-2638, 2006.

4. Williams AH, Liu N, van Rooij E and Olson EN: MicroRNA control of muscle development and disease. Curr Opin Cell Biol 21: 461-469, 2009.

5. Bartel DP: MicroRNAs: Genomics, biogenesis, mechanism and function. Cell 116: 281-297, 2004.

6. Wang F, Niu G, Chen X and Cao F: Molecular imaging of microRNAs. Eur J Nucl Med Mol Imaging 38: 1572-1579, 2011.

7. Goljanek-Whysall K, Sweetman D and Munsterberg AE: MicroRNAs in skeletal muscle differentiation and disease. Clin Sci (Lond) 123: 611-625, 2012.

8. Rao PK, Kumar RM, Farkhondeh M, Baskerville S and Lodish HF: Myogenic factors that regulate expression of muscle-specific microRNAs. Proc Natl Acad Sci USA 103: 8721-8726, 2006.

9. Rosenberg MI, Georges SA, Asawachaicharn A, Analau E and Tapscott SJ: MyoD inhibits Fstl1 and Utrn expression by inducing transcription of miR-206. J Cell Biol 175: 77-85, 2006.

10. Chen JF, Mandel EM, Thomson JM, Wu Q, Callis TE, Hammond SM, Conlon FL and Wang DZ: The role of microRNA-1 and microRNA-133 in skeletal muscle proliferation and differentiation. Nat Genet 38: 228-233, 2006.

11. Kim HK, Lee YS, Sivaprasad U, Malhotra A and Dutta A: Muscle-specific microRNA miR-206 promotes muscle differentiation. J Cell Biol 174: 677-687, 2006.

12. Knezevic I, Patel A, Sundaresan NR, Gupta MP, Solaro RJ, Nagalingam RS and Gupta M: A novel cardiomyocyte-enriched microRNA, miR-378, targets insulin-like growth factor 1 receptor: Implications in postnatal cardiac remodeling and cell survival. J Biol Chem 287: 12913-12926, 2012.

13. Megiorni F, Cialfi S, McDowell HP, Felsani A, Camero S, Guffanti A, Pizer B, Clerico A, De Grazia A, Pizzuti A, et al: Deep Sequencing the microRNA profile in rhabdomyosarcoma reveals down-regulation of miR-378 family members. BMC Cancer 14: 880, 2014.

14. Huang da W, Sherman BT and Lempicki RA: Systematic and integrative analysis of large gene lists using DAVID bioinformatics resources. Nat Protoc 4: 44-57, 2009.

15. Shimada A: PCR-based site-directed mutagenesis. Methods Mol Biol 57: 157-165, 1996.

16. Hupkes M, Sotoca AM, Hendriks JM, van Zoelen EJ and Dechering KJ: MicroRNA miR-378 promotes BMP2-induced osteogenic differentiation of mesenchymal progenitor cells. BMC Mol Biol 15: 1, 2014.

17. Creighton CJ, Nagaraja AK, Hanash SM, Matzuk MM and Gunaratne PH: A bioinformatics tool for linking gene expression profiling results with public databases of microRNA target predictions. RNA 14: 2290-2296, 2008.

18. Szklarczyk D, Franceschini A, Kuhn M, Simonovic M, Roth A, Minguez P, Doerks T, Stark M, Muller J, Bork Peer, et al: The STRING database in 2011: Functional interaction networks of proteins, globally integrated and scored. Nucleic Acids Res 39: D561-568, 2011.

19. Zhao Y, Samal E and Srivastava D: Serum response factor regulates a muscle-specific microRNA that targets Hand2 during cardiogenesis. Nature 436: 214-220, 2005.

20. Lagos-Quintana M, Rauhut R, Yalcin A, Meyer J, Lendeckel W and Tuschl T: Identification of tissue-specific microRNAs from mouse. Curr Biol 12: 735-739, 2002.

21. McCarthy JJ: MicroRNA-206: The skeletal muscle-specific myomiR. Biochim Biophys Acta 1779: 682-691, 2008.

22. Eisenberg I, Eran A, Nishino I, Moggio M, Lamperti C, Amato AA, Lidov HG, Kang PB, North KN, Mitrani-Rosenbaum S, et al: Distinctive patterns of microRNA expression in primary muscular disorders. Proc Natl Acad Sci USA 104: 17016-17021, 2007.

23. Cacchiarelli D, Martone J, Girardi E, Cesana M, Incitti T, Morlando M, Nicoletti C, Santini T, Sthandier O, Barberi L, et al: MicroRNAs involved in molecular circuitries relevant for the Duchenne muscular dystrophy pathogenesis are controlled by the dystrophin/nNOS pathway. Cell Metab 12: 341-351, 2010.

24. Liu N, Williams AH, Kim Y, McAnally J, Bezprozvannaya S, Sutherland LB, Richardson JA, Bassel-Duby R and Olson EN: An intragenic MEF2-dependent enhancer directs muscle-specific expression of microRNAs 1 and 133. Proc Natl Acad Sci USA 104: 20844-20849, 2007.

25. Feng Y, Cao JH, Li XY and Zhao SH: Inhibition of miR-214 expression represses proliferation and differentiation of $\mathrm{C} 2 \mathrm{C} 12$ myoblasts. Cell Biochem Funct 29: 378-383, 2011. 
26. Sun Q, Zhang Y, Yang G, Chen X, Zhang Y, Cao G, Wang J, Sun Y, Zhang P, Fan M, et al: Transforming growth factor-beta-regulated miR-24 promotes skeletal muscle differentiation. Nucleic Acids Res 36: 2690-2699, 2008.

27. Wong CF, and Tellam RL: MicroRNA-26a targets the histone methyltransferase Enhancer of Zeste homolog 2 during myogenesis. J Biol Chem 283: 9836-9843, 2008.
28. Hou X, Tang Z, Liu H, Wang N, Ju H and Li K: Discovery of MicroRNAs associated with myogenesis by deep sequencing of serial developmental skeletal muscles in pigs. PLoS One 7: e52123, 2012.

29. Gagan J, Dey BK, Layer R, Yan Z and Dutta A: MicroRNA-378 targets the myogenic repressor MyoR during myoblast differentiation. J Biol Chem 286: 19431-19438, 2011. 